Experimentally, the electrocardiographic observation were carried out using 12 mongreal dogs. A baloon was inserted into the subdural space in 8 cases, and in 4 cases cerebral contusion were conducted. At the infusion of air, sinus bradicardia, suppression of sinus and atrio-ventricular nodes was always noted and in some cases nodal escaped beat was recognized. And these changes were recovered immediately after the air was released. Otherwise, such change as frequent occurrence of extrasystole, supraventricular tachycardia and abnormality in ST-T were confirmed.

\title{
43. A Contribution to the Mechanism of Facial Twitches Produced by Electrical Stimulation of the Amygdala
}

\author{
Shoji Salto and Mitsukuni MUrasaki \\ Inokashira Mental Hospital \\ Tsunekatsu Hara and Toshio Hara \\ Dept. of Neuropsychiatry, Keio University
}

In the preceding report, the emergence of rhythmic sharp waves, considered as evidence of after-discharges elicited by electrical stimulation of the amygdaloid nuclei, were proved to be synchronous with ipsilateral facial twitches in cats. The observation resulted in conclusion that the facial cramps occured in "psychomotor seizures" might have their origin in the amygdala.

In subsequent series of experiment to clarify the process of discharges evoking facial phenomena, the results obtained so far are summarized are follows.

Ipsilateral and/or contralateral ablation of the cerebral cortex including sensory-motor areas caused no change on the facial twitches.

Though the experimental lesions in the ipsilateral dorsomedial nucleus of the thalamus did not elicit and facial twitches, the after-discharges were observed evidently on the amygdaloid electrodes. On the contrary, the stimulation of the similar thalamic nucleus produced ipsilateral facial twitches, whereas the stimulation of other thalamic nuclei did not present the said phenomenon.

Basing upon the above mentioned data, also supported by the results of our collaborators, the nucleus medialis dorsalis of the thalamus seems to be responsible as the relay nucleus of the impulse from the amygdala to the facial motor nerve. 